\title{
Strong reduction of laser power noise by means of a Kerr nonlinear cavity
}

\author{
Alexander Khalaidovski, André Thüring, Henning Rehbein, Nico Lastzka, Benno Willke, \\ Karsten Danzmann, and Roman Schnabel* \\ Max-Planck-Institut für Gravitationsphysik (Albert-Einstein-Institut) and Institut für Gravitationsphysik, Leibniz Universität Hannover, \\ Callinstr. 38, 30167 Hannover, Germany
}

(Received 3 June 2009; published 2 November 2009)

\begin{abstract}
We demonstrated the power noise reduction of a continuous-wave laser field by means of an effective third-order Kerr nonlinear cavity. In contrast to conventional noise reduction schemes relying on linear cavities, a strong noise suppression at Fourier frequencies below the linewidth of the nonlinear cavity was possible. The laser light was reflected off a Kerr nonlinear cavity that had a half width half maximum linewidth of 4.5 MHz. The cavity was operated slightly off-resonance at approximately half of the maximum power buildup, close to its so-called critical state; a power noise reduction of up to $32 \mathrm{~dB}$ at Fourier frequencies below $1 \mathrm{MHz}$ was observed after reflection. The effective third-order nonlinearity was a so-called cascaded second-order nonlinearity of $\mathrm{MgO}: \mathrm{LiNbO}_{3}$. The laser had a power of $0.75 \mathrm{~W}$ at the wavelength of $1064 \mathrm{~nm}$.
\end{abstract}

DOI: 10.1103/PhysRevA.80.053801

PACS number(s): 42.65.Hw, 42.65.Ky, 42.50.-p, 42.79.-e

\section{INTRODUCTION}

An active laser power stabilization is essential for many high-precision optics experiments, since free-running laser power fluctuations are often limiting the sensitivity of these experiments. Passive noise filtering provided by the transmission through optical cavities can efficiently be used in order to reduce power fluctuations at Fourier frequencies above the cavity linewidth. More adequate in most cases are active feedback control circuits. Here, a certain fraction of the laser power is detected by photodiodes in order to sense the power fluctuations and different kinds of actuators such as acousto-optical modulators are then used to reduce the laser noise on the residual beam. Especially in applications that require high power stability at low frequencies, a constraint arises for active stabilization schemes, which is caused by (presumably photodiode internal) unknown noise sources associated with the large photocurrent. At $10 \mathrm{~Hz}$, this photodiode noise already exceeds the shot noise of a 100 $\mathrm{mW}$ laser beam which corresponds to a relative power noise of approximately $2 \times 10^{-9} \mathrm{~Hz}^{-1 / 2}$ [1]. At just slightly lower relative power noise levels (corresponding to slightly higher powers), an additional problem arises and the sensing sensitivity would be limited by the available dynamic range of photodiodes and their amplifiers. Hence, novel schemes are required that reduce the required dynamic range as well as the detected power of the sensing device [2]. But again, these schemes are typically limited to frequencies comparable or above the linewidth of the optical cavity involved.

Another way out, proposed quite a while ago, bases on the optical Kerr effect. It does not require any photodetection $[3,4]$ and can be combined with optical cavities in order to yield a power noise reduction by self-modulation [5]. Here, the name optical Kerr effect refers to the intensity-dependent phase shift during propagation through a (third-order) nonlinear medium. One problem of this approach is the typically rather low third-order nonlinearity of high-quality optical

\footnotetext{
*roman.schnabel@aei.mpg.de
}

materials. In the pulsed laser regime, high laser intensities can be used to compensate for this and strong noise reductions of certain field quadratures even below the shot noise, i.e., into the nonclassical regime of squeezed states, were demonstrated [6,7].

In the continuous-wave laser regime, an intensity increase through tight focusing and power buildups in optical resonators are typically not sufficient and materials with higher third-order nonlinearities are desired. A promising solution is the use of interacting $\chi^{(2)}$ nonlinearities as proposed for the first time already 4 decades ago [8]. However, up to now, no strong noise suppression of continuous-wave light had been demonstrated. In [9], the classical noise of a $\mathrm{cw}$ laser beam of less than $30 \mathrm{~mW}$ power was reduced by $1.5 \mathrm{~dB}$. In [10], the more demanding regime of nonclassical states was targeted and squeezed states of a $0.45 \mathrm{~mW} \mathrm{cw}$ beam were observed. The measured noise reduction was 30\% (approximately $1.5 \mathrm{~dB}$ ) below the vacuum noise level.

In this paper, we report on the observation of a strong noise reduction by more than $32 \mathrm{~dB}$ of a commercial lownoise, continuous-wave, solid-state Nd:yttrium aluminum garnet (YAG) laser of $0.75 \mathrm{~W}$ by means of an off-resonant (detuned) Kerr nonlinear cavity. The Kerr nonlinear cavity was a detuned, single-ended, standing-wave cavity containing a magnesium-oxide-doped lithium-niobate $\left(\mathrm{MgO}: \mathrm{LiNbO}_{3}\right)$ crystal. The noise reduction was observed by photoelectric detection with a single photodiode.

\section{OPTICAL KERR EFFECT AND NOISE REDUCTION}

In this section, we review the optical Kerr effect induced transformation of a single-mode optical field including (classical) noise. We consider the electrical field of a laser beam passing a third-order nonlinear medium at some specific position. We can then write the electric field with an optical carrier frequency $\omega_{0}$ in terms of the complex amplitudes $A, A^{*}$,

$$
E(t) \sim A(t) e^{-i \omega_{0} t}+A^{*}(t) e^{i \omega_{0} t} .
$$

It is convenient to decompose the complex amplitudes into their expectation value $\bar{A}$ and a noise term $\delta A$, 


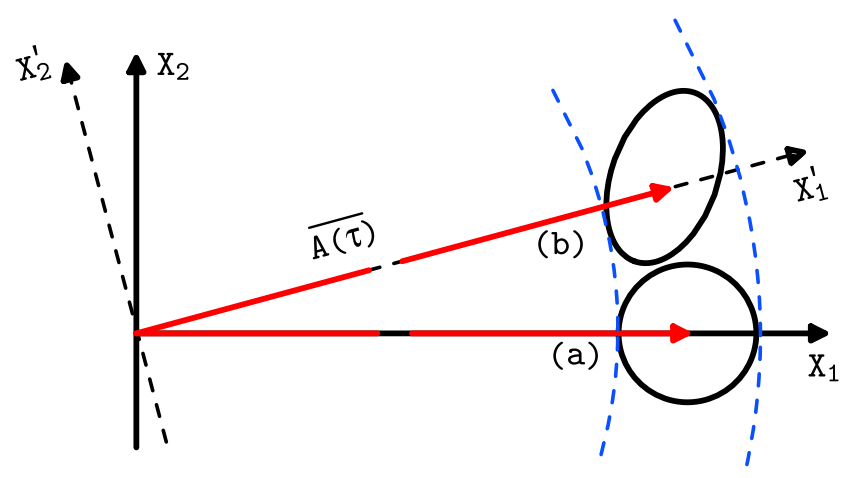

FIG. 1. (Color online) Phase space description of a light field (a) before and (b) after the interaction with an optical Kerr medium. $X_{1,2}$ denote the amplitude and the phase quadratures. Due to the intensity-dependent phase shift, the noise distribution is transformed into an elliptic shape.

$$
A(t)=\bar{A}+\delta A(t) \quad \text { with } \quad \overline{\delta A(t)}=0,
$$

where $\sqrt{\delta A(t)^{2}}$ corresponds to the standard deviation of $A(t)$.

We further decompose $A(t)$ and $\delta A(t)$ into their real and imaginary parts

$$
A(t)=X_{1}(t)+i X_{2}(t), \quad \delta A(t)=\delta X_{1}(t)+i \delta X_{2}(t),
$$

where $X_{1}(t), X_{2}(t)$ and $\delta X_{1}(t), \delta X_{2}(t)$ provide a real-valued phase-space description of the laser field and are its (noise) amplitude and phase quadratures, respectively. In the following, we also omit the time dependence but consider the interaction time with the Kerr medium $\tau$. When the field propagates through the Kerr medium, at the output the complex amplitude reads (cf., e.g., Ref. [11])

$$
A(\tau)=e^{-i \chi^{(3)} A^{*} A \tau} A(0),
$$

where $\chi^{(3)}$ is proportional to the third-order nonlinear susceptibility. Keeping just first-order terms of $\delta A$, we obtain from Eq. (4)

$$
\begin{aligned}
& A(\tau)=e^{-i \chi^{(3)}|\bar{A}|^{2} \tau}\left\{\left[\left(1-i \chi^{(3)} \tau|\bar{A}|^{2}\right) \delta A(0)-i \chi^{(3)} \tau \bar{A}^{2} \delta A^{*}(0)\right]\right. \\
& +\bar{A}\} \text {. }
\end{aligned}
$$

The underlying calculation is carried out in detail by Shirasaki and Haus in Ref. [12]. When converting Eq. (5) into an expression for the quadratures, one can readily deduce that the Kerr medium transforms the phase-space representation of the light field. First of all, the Kerr effect results in a phase-space rotation of $\overline{A(\tau)}$. Second, which is more interesting, the noise distribution is also transformed. Assuming a circular and Gaussian phase-space distribution of the quadrature input noise, i.e., the input quadratures have uncorrelated noise of the same strength, the Kerr-effecttransformed output noise corresponds to an ellipse (see Fig. 1). Roughly speaking, the top of the initial noise circle corresponds to a higher intensity and therefore is further phase shifted by the Kerr effect than the bottom. As the interesting consequence, the ellipse's semiminor axis is smaller than the circle's diameter. For small rotations, the noise distribution remains Gaussian and it turns out that the noise projection on the axis in direction of $\overline{A(\tau)}$ always remains unchanged. However, the noise projection on a certain linear combination of the quadratures $X_{1}(t)$ and $X_{2}(t)$ is smaller than any projection of the input noise. We emphasize that in this work we are interested in the Kerr noise reduction of precisely the length of $\overline{A(\tau)}$. Such a noise reduction is indeed possible by an additional differential phase-space rotation by other means (see Sec. IV).

Note that the considerations made here can be readily converted into a quantum-mechanical description of the Kerr effect. In quantum theory, the complex amplitudes are associated with mutually adjoint operators. These operators correspond to the annihilation and creation Heisenberg operators and obey the well-known boson commutation relations. Furthermore, it should be noted that we have focused on a linearized Kerr transformation, which is valid for the experimental setup investigated in this paper. The linearized formalism might break down for large rotations and hence a non-Gaussian noise distribution (Wigner function) can occur [4].

\section{CASCADED KERR NONLINEARITY}

In order to exploit the optical Kerr effect for laser noise reduction, a high-quality third-order nonlinear optical material is required. Typical third-order nonlinearities of optical media are, unfortunately, rather small. In the pulsed laser regime, high laser intensities can be used to compensate for this.

In the continuous-wave laser regime, interacting $\chi^{(2)}$ nonlinearities can be employed to obtain an effective Kerr effect. It was indeed shown that by the means of second-harmonic generation (SHG) and a subsequent frequency downconversion, an intensity dependence of the refractive index can be realized. Such a scheme represents an effective thirdorder nonlinear Kerr medium but utilizes the second-order nonlinearity of, for example, lithium niobate or KTP (potassium titanyl phosphate, $\mathrm{KTiOPO}_{4}$ ) [13]. The lower order of nonlinearity results in a comparatively strong effective thirdorder nonlinearity.

Interacting nonlinearities have been discussed analytically and numerically since the first days of nonlinear optics $[8,9,14-16]$. Here, we summarize the results focusing on the physical understanding of the mechanism that leads to a cascaded Kerr nonlinearity and hence to a noise suppression. For this purpose, we consider a lossless second-order nonlinear material and the second-harmonic generation in such a material of length $L$, driven by a field of fundamental frequency $\omega_{0}$. In case of optimum phase matching between the fundamental and the second-harmonic mode of the EM field, the second-harmonic light generated along the length $L$ interferes constructively only with itself over the whole interaction length. Now let us assume that the phase matching is artificially worsened until the first conversion minimum is reached. The new situation can be conceptually understood by splitting the crystal in two parts of lengths $L / 2$. In both parts, when considered independently, SHG still occurs, although this process is now no longer optimized anymore. However, the contributions of both parts now exhibit a dif- 
ferential phase shift. This phase shift leads to a completely destructive interference after the full crystal length $L$. Consequently, no light at frequency $2 \omega_{0}$ exits the crystal. The fundamental field exiting the crystal can be thought of as a composition of two parts. One part represents the field that previously has experienced up-conversion to the secondharmonic frequency. The second part represents the field that never experienced up-conversion. The phases of the two parts are different because the phase matching is not optimized as said before and the refractive indices at the two wavelengths are therefore different. Since up-conversion is a nonlinear $\left(\chi^{(2)}\right)$ process, the two parts change their weights if the input intensity changes. Effectively, the transmitted fundamental light experiences an intensity-dependent phase shift formally identical to the optical Kerr effect observed in a $\chi^{(3)}$ medium.

A simple mathematical description is obtained by solving the nonlinear wave equation for the involved modes of the optical field assuming a weak conversion of the fundamental field (fixed intensity approximation) [17]. In this case, we obtain the expression

$$
\begin{aligned}
\phi(z)= & \phi_{\text {prop }}(z)+\frac{\Delta k z}{8+\frac{(\Delta k)^{2}}{\chi^{\prime} I_{\text {fund }}}} \\
& \times\left\{1-\operatorname{sinc}\left[z \sqrt{\left(\frac{\Delta k}{2}\right)^{2}+2 \chi^{\prime} I_{\text {fund }}}\right]\right\},
\end{aligned}
$$

describing the phase dependence of the fundamental field on the coordinate of propagation, $z$. Here, $\Delta k$ is the wave vector mismatch between the fundamental and the second-harmonic waves, $\phi_{\text {prop }}$ is the intensity-independent phase shift due to propagation, while $\chi^{\prime}$ contains material-specific information (as the effective nonlinearity and the propagation speed inside the crystal) and further depends on both frequencies involved. In the case of minimal conversion efficiency (at the first phase matching minimum with $\Delta k \approx 2 \pi / z$ ), this expression simplifies to

$$
\phi(z) \approx \phi_{\text {prop }}(z) \pm \frac{\chi^{\prime}}{2 \pi} z^{2} I_{\text {fund }},
$$

whereas in the case $\Delta k=0$, the intensity dependence vanishes. This fact allows us to switch between pure frequency doubler and pure Kerr-like behavior through a simple adjustment to the corresponding phase matching condition (i.e., using the temperature dependence of the refractive index in birefringent crystals).

\section{CAVITY PHASE-SPACE ROTATION}

A single-mode optical field that has passed a Kerr medium does not show any reduction of its power noise (Sec. II). In order to observe a reduction of power noise, a differential phase-space rotation between the noise and the expectation value of the complex amplitude that represents the single-mode carrier field is required. In other words, the semiminor axis of the noise ellipse needs to be aligned in direction of the complex phasor in Fig. 1. Such a rotation can be provided by reflecting the beam off a detuned, but standard optical cavity (without any nonlinear medium). This scheme, based on the dispersion of the cavity, was proposed in order to support quantum nondemolition measurements in Ref. [18] and was demonstrated for squeezed states in Ref. [19]. Since no additional field needs to be mixed with the input beam, no optical loss occurs. However, the phase-space rotation depends on the Fourier frequency and therefore can be optimized only for a certain Fourier frequency component of the quadrature phase-space spanned by $X_{1}(\Omega, \Delta \Omega, t)$ and $X_{2}(\Omega, \Delta \Omega, t)$, where $\Omega$ is the Fourier frequency and $\Delta \Omega$ the resolution bandwidth. We note that these quantities describe amplitude and phase modulations of our laser beam, respectively, and therefore pairs of upper and lower sideband fields. However, they can also be seen as the spectrally bandpassfiltered counterparts of $X_{1,2}(t)$ as defined in Eq. (3). In the following, we summarize the connection between phasespace rotation in Fourier space, linewidth of the rotating cavity, and cavity detuning in view of our experiment in which we combined the Kerr noise reduction and an optimized phase-space rotation in a single optical device, a detuned Kerr nonlinear cavity.

To comprehend the phase-space rotation of a light field in reflection of a detuned cavity, one has to account for its frequency dependent phase shift $\psi(\Omega)$. For simplicity, we assume the considered light field to be just modulated in its amplitude quadrature and a loss-free single-ended cavity (the end mirror has a reflectivity of $\rho_{2}=1$ ) so that the reflectivity of the cavity is unity for all frequencies. Accordingly, any phase-space rotation can be traced back to the phase shift given by

$$
\psi(\Omega)=\arg \left(\frac{\rho_{1}-e^{2 i(\phi+\Omega L / c)}}{1-\rho_{1} e^{2 i(\phi+\Omega L / c)}}\right) .
$$

Here, $\phi$ denotes the detuning related to the carrier frequency $\omega_{0}, c$ the speed of light, and $L$ the cavity length. Notice that $\omega_{0} L / c$ corresponds to the detuning $\phi=0$.

From Eq. (8), we immediately see that for Fourier frequencies within the half width half maximum (HWHM) cavity linewidth $(\Omega \ll \gamma)$, only marginal phase-space rotations occur. For frequencies far above the cavity linewidth, the opposite situation can occur and strong phase-space rotations are possible. Let us assume $\Omega \gg \gamma$ and a cavity detuning such that solely the upper sideband resonates $(-\phi=+\Omega L / c)$. In this case, the phase shifts of the lower sideband and the carrier field are negligible, whereas the upper sideband gets phase shifted by $-180^{\circ}$. As described in [19], the quadrature angle then rotates by half of this value, i.e., by $-90^{\circ}$, and the initial amplitude quadrature (amplitude modulation) is rotated into the phase quadrature (phase modulation) and vice versa. This rotation value holds only for a rather small bandwidth and, for example, for sideband frequencies $\Omega \pm \gamma$, the rotation is just $\mp 45^{\circ}$. It turns out that for large Fourier frequencies, arbitrary phase-space rotations can be achieved, but not for small frequencies inside the HWHM cavity linewidth. In contrast to that, it was then shown in Ref. [5] that a detuned cavity containing a Kerr medium cannot only provide a noise reduction via the Kerr effect, but can indeed simultaneously provide the required additional phase-space 


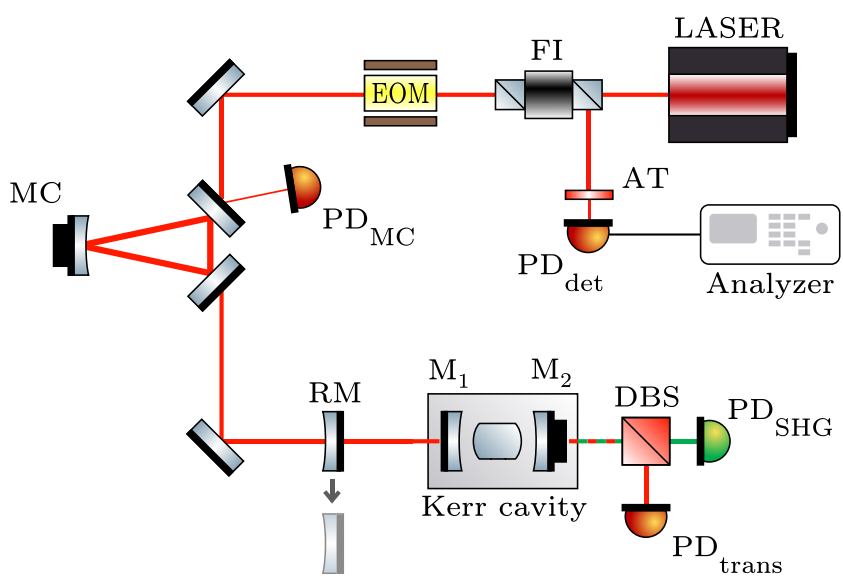

FIG. 2. (Color online) Schematic of the experimental layout. FI: Faraday isolator, EOM: electro-optical modulator, MC: mode cleaner cavity, PD: photodetector, RM: mode-matched reference mirror, DBS: dichroic beam splitter, AT: variable attenuator, and Analyzer: spectrum analyzer. Details are described in the text.

rotation for frequencies inside the cavity linewidth in order to achieve a laser power noise reduction of a reflected laser beam.

\section{EXPERIMENT}

In our experiment, we demonstrated the operation of a detuned Kerr nonlinear cavity that was able to significantly reduce the noise power of a laser beam. Such a device is possible if the interplay between Kerr transformation (Sec. III) and cavity detuning (Sec. IV) is optimized, as theoretically analyzed in Ref. [5].

The simplified experimental layout is shown in Fig. 2. The system is driven by a monolithic nonplanar Nd:YAG ring laser (Mephisto 2000, InnoLight) with up to $2 \mathrm{~W}$ output power. This source provides a low-noise continuous-wave single-mode single-frequency laser beam at $1064 \mathrm{~nm}$ with a very clean spatial beam profile with typically less than $2.5 \%$ of the power in higher-order modes. The relative power noise is below $1 \times 10^{-6} \mathrm{~Hz}^{-1 / 2}$ for Fourier frequencies $f=\Omega / 2 \pi$ above $30 \mathrm{~Hz}$ and shows a peak approximately $40 \mathrm{~dB}$ high at the frequency corresponding to the laser relaxation oscillation at frequencies around $1 \mathrm{MHz}$. Above these frequencies, the power noise falls with $1 / f^{2}$ and is less than $3 \mathrm{~dB}$ above the shot noise of $100 \mathrm{~mW}$ for frequencies above $10 \mathrm{MHz}$. A detailed characterization of eight similar lasers can be found in $[20]$.

The laser beam was first transmitted through a Faraday isolator and a mode cleaner ring cavity with a finesse of $\mathcal{F}$ $\approx 500$ and a half width half maximum linewidth of approximately 1.5 MHz. Besides working as a low-pass filter, the cavity also acts as a spatial filter; it was held on resonance with the $1064 \mathrm{~nm}$ laser beam via a Pound-Drever-Hall (PDH) locking scheme [21]. The filtered beam had a power of $0.75 \mathrm{~W}$ and was mode matched into our Kerr nonlinear cavity with an efficiency of $99 \%$. The beam with changed noise performance was then retroreflected and passed the mode cleaner a second time. The Faraday rotator sent the full

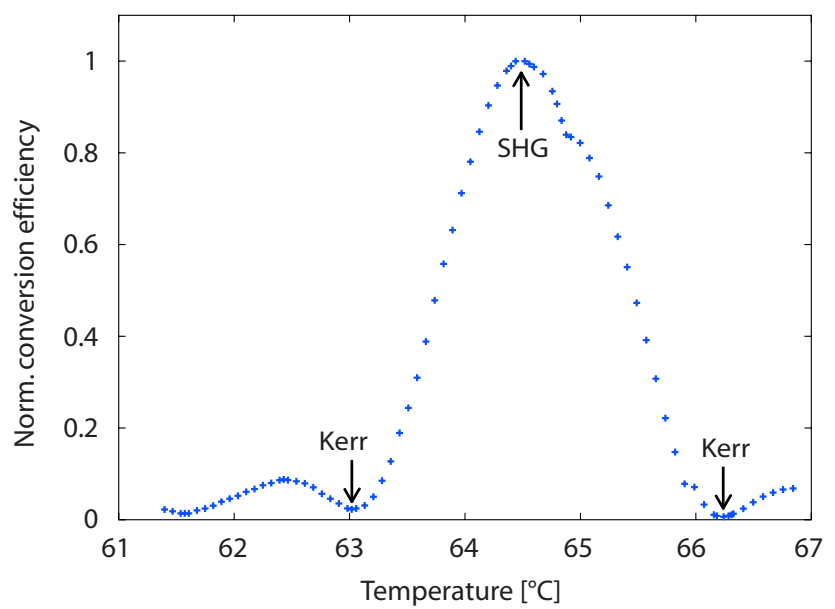

FIG. 3. (Color online) Measured second-harmonic generation (SHG) conversion efficiency vs crystal temperature (input laser power $P_{\mathrm{TEM}_{00}}=95 \mathrm{~mW}$ ). The temperature was measured via a thermoresistor. The absolute values could be calculated with an accuracy of $\pm 1{ }^{\circ} \mathrm{C}$. The Kerr effect can be observed in conversion minima slightly offset from the optimum SHG temperature: $\left(T_{\text {Kerr,lower }}-T_{\text {SHG }}\right)=-1.41^{\circ} \mathrm{C}$ and $\left(T_{\text {Kerr,upper }}-T_{\text {SHG }}\right)=+1.77{ }^{\circ} \mathrm{C}$.

power toward a single photodiode, where the beam was detected after attenuation and eventually analyzed with a spectrum analyzer.

The Kerr cavity had a HWHM linewidth of $\gamma$ $\approx 4.5 \mathrm{MHz}$ and consisted of a $1.5 \times 2.0 \times 6.3 \mathrm{~mm}^{3}$ $7 \% \mathrm{MgO}: \mathrm{LiNbO}_{3}$ crystal placed in between two curved mirrors of $25 \mathrm{~mm}$ radius of curvature. The coupling mirror had the reflectivities $R_{\mathrm{M}_{1}}(1064 \mathrm{~nm})=0.983$ at the fundamental and $R_{\mathrm{M}_{1}}(532 \mathrm{~nm})=0.01$ at the generated second harmonic, whereas the end mirror was highly reflective at 1064 $\mathrm{nm}$ and weakly reflective at $532 \mathrm{~nm}\left[R_{\mathrm{M}_{2}}(532 \mathrm{~nm})=0.02\right]$. The intracavity crystal surfaces were antireflective coated $\left(R_{\mathrm{AR}}=0.001\right)$ at both 1064 and $532 \mathrm{~nm}$. The crystal was separated from the mirrors by $23.6 \mathrm{~mm}$ air gaps, creating a cavity mode for the resonant fundamental light with a $27 \mu \mathrm{m}$ waist at the center of the crystal. Together with the round-trip loss that was estimated to $0.5 \%$, the cavity finesse resulted in a value of $\mathcal{F} \approx 274$. For cavity characterization and SHG, the cavity length was held on resonance using another PDH locking loop. To investigate the phase matching of the Kerr cavity, the second-harmonic power was recorded as a function of crystal temperature. Figure 3 shows the normalized phase matching curve for a $\mathrm{TEM}_{00}$ input mode for crystal temperatures between 61 and $67{ }^{\circ} \mathrm{C}$. As expected, the curve in Fig. 3 is well described by a sinc function. The additional peak at a temperature of about $64.9{ }^{\circ} \mathrm{C}$ is most likely due to the nonzero reflectivity of the cavity mirrors at $532 \mathrm{~nm}$ and its effect on the differential phase between the reflected fields at 1064 and $532 \mathrm{~nm}$ [22]. At the SHG operation point of our nonlinear cavity, we observed maximum conversion efficiency of $\approx 72 \%$.

In order to achieve Kerr-effect power noise suppression, the crystal temperature was tuned to one of the two first conversion minima (Fig. 3). At these crystal temperatures, the cavity exhibited a multistable behavior as is characteristic for a $\chi^{(3)}$ medium. Figure 4 shows the observed asymme- 


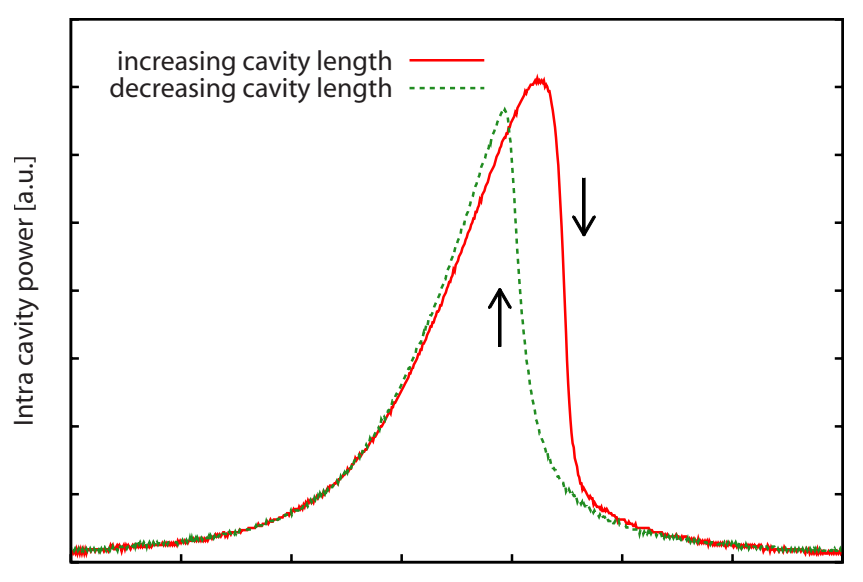

Cavity length [a.u.]

FIG. 4. (Color online) Line shapes of the resonator mode recorded in transmission. The cavity was operated at the upper temperature Kerr point (see Fig. 3). The hysteresis clearly demonstrates the presence of an intensity-dependent phase shift and hence a strong cascaded nonlinearity. An equivalent hysteresis, but with "flipped" asymmetry, was also observed in the other Kerr point (not displayed), as expected for opposite signs of the nonlinear phase shift. The incident fundamental power was approximately $780 \mathrm{~mW}$.

try of the Airy peak of our Kerr nonlinear cavity when the cavity length was increased or decreased, respectively. Any thermal effects were suppressed by a short scanning time through resonance of $\approx 1 \mu \mathrm{s}$. We experimentally verified that the asymmetry's orientation depended on the sign of the effective nonlinearity and thus on the phase matching minimum chosen (Fig. 3). The difference in the two curves shown in Fig. 4 is due to the intensity-dependent phase shift in our nonlinear cavity. Please note that the observation of a Kerr effect hysteresis on a scanned cavity is not a sufficient proof for a multistable regime because of the finite cavity loading time. The shown measurement, together with a numerical model, allows us to estimate that the multistable regime is entered at an input power of approximately $800 \mathrm{~mW}$. The two curves in Fig. 4 were measured with $780 \mathrm{~mW}$ and therefore represent a situation very close to the critical state of the cavity in which the slope of the Airy peak should be infinitely steep on one side. Such behavior can in principle be observed, but only at very slow scanning speeds. In practice, the scanning speed must be sufficiently high so as to minimize the impact of acoustic and thermal effects. For higher speeds, the loading time of the cavity comes into play and a vertical slope can practically not be observed.

Figure 5 shows the measurement results that demonstrate the laser power noise reduction by means of our Kerr nonlinear cavity. All curves describe the noise spectrum of a 0.75 $\mathrm{W}$ laser beam and were detected with photodiode $\mathrm{PD}_{\text {det. }}$. The top peaked curve shows the laser's initial power noise after two transmissions through the mode cleaner MC measured using the flip mirror as shown in Fig. 3. The other curves show the power noise spectra of the same laser when being reflected off our detuned Kerr cavity and when the operation point on the steep slope of the Airy peak (Fig. 4) was varied and stabilized by an appropriate dc offset lock. The reflected laser power was attenuated to exactly the same reference

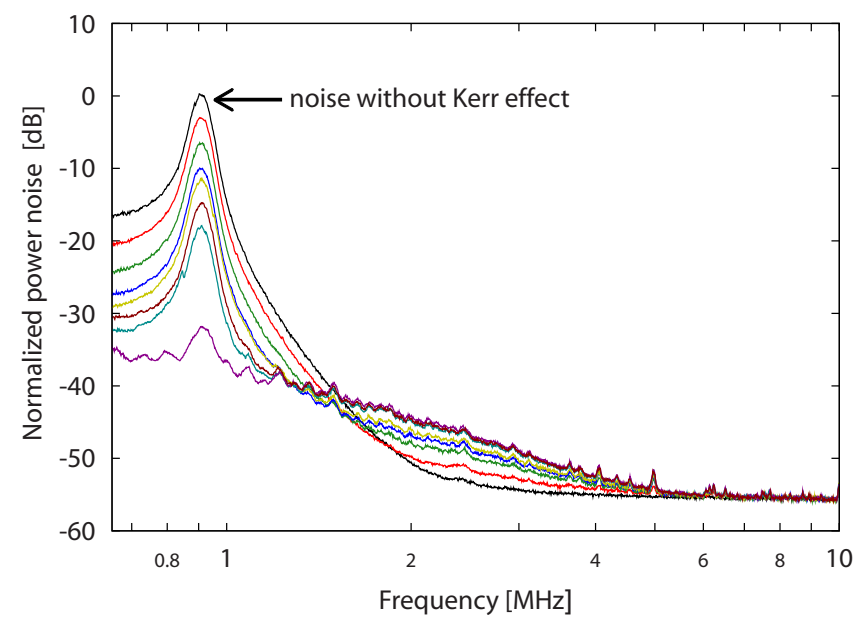

FIG. 5. (Color online) Reflected fundamental power noise vs Fourier frequency for laser beams of identical dc power. The top peaked curve shows the laser's initial power noise after two transmissions through the mode cleaner MC, measured using the modematched reference mirror as shown in Fig. 3. The other curves show the noise on the beam reflected at the Kerr nonlinear cavity locked on several states on the steep resonance slope. The maximum amplitude noise reduction (at about $0.9 \mathrm{MHz}$ ) was achieved at a detuning where the internal cavity field reaches approximately $3 / 4$ of the maximum buildup obtained at resonance.

value and was detected by the photodiode. A maximum noise suppression of $32 \mathrm{~dB}$ was observed slightly below $1 \mathrm{MHz}$ for a Kerr cavity operation point at about 3/4 of the Airy peak's maximum. All curves in Fig. 5 were shot noise limited at frequencies above $9 \mathrm{MHz}$. At intermediate frequencies, they showed additional noise that increased with an increasing Kerr effect and probably arose from internal Brillouin scattering.

At Fourier frequencies below $600 \mathrm{kHz}$, our setup showed nonstationary noise and the measurement of a family of traces with varying Kerr-operation points was not reproducible. However, we anticipate that the observed noise reduction will also be observable down to audio-band frequencies if acoustic vibrations, air turbulence, and dust particle flows are sufficiently reduced.

\section{DISCUSSION}

In our experiment, we investigated the power noise suppression of a $0.75 \mathrm{~W}$ single-frequency continuous-wave laser beam at a wavelength of $1064 \mathrm{~nm}$ and observed a maximum suppression of $32 \mathrm{~dB}$. We performed several experiments in order to confirm that the observed noise suppression was due to the optical Kerr effect. When we changed the crystal temperature and thereby moved the cavity from the upper to the lower conversion minimum, we observed the Kerr nonlinearity to change its sign, as predicted from theory. In the actual measurement, we observed that the steep slope, as in Fig. 4, changed from right to left without changing the scanning direction of the cavity length. When the cavity was locked to points on the steep slope, the observed maximum noise suppression was independent merely of the choice of the two 
conversion minima, but always dependent on the input power, as predicted from theory. For powers above approximately $0.8 \mathrm{~W}$, the cavity was in its multistable regime; for smaller powers, the noise suppression degraded rapidly.

The curves in Fig. 5 show the power noise spectra for different locking points of the Kerr cavity. As we are interested only in the power noise reduction between different experimental situations (and not in the absolute values of the power noise), we took the approach to simply plot the $\mathrm{dB}$ value given by the spectrum analyzer. This value is proportional to $20 \log _{10}\left[\delta P(\Omega, \Delta \Omega) / P_{\text {ref }}\right]$ with the power fluctuations $\delta P$ being proportional to $|\bar{A}| \sqrt{\left[X_{1}(\Omega, \Delta \Omega, t)\right]^{2}}$, with $X_{1}$ being the real part of $A$ according to Eq. (3). The $\mathrm{dB}$ value corresponds to the noise spectral density integrated over a fixed frequency range normalized to a fixed reference voltage. The knowledge neither of the frequency band nor of the reference voltage is of any relevance for our discussion. The same holds for the frequency-dependent response function of the photodetector as we do not compare noise levels for different frequencies to each other. However, we note that the detector's response function had a white spectrum within the frequency band plotted and its dark noise was well below the noise recorded.

We finally note that, although we observed an amplitude quadrature noise suppression of $32 \mathrm{~dB}$, we were still far above the shot noise limit. The reason was that generally high laser powers at low frequencies (around and below the laser relaxation oscillation) typically carry an enormous amount of classical noise. The goal of this work was the reduction of this classical noise, for instance, in view of an application in gravitational-wave detectors. In order to beat the shot noise limit for continuous-wave radiation, one has to consider Fourier frequencies far above the laser's relaxation oscillation. Additionally, the input power has to be reduced in order to get closer to the shot noise limit from the very beginning: a reduction of input power then requires materials with stronger nonlinearities, for example, periodically poled second-order nonlinear crystals, and a tighter focusing.

\section{CONCLUSIONS}

It was shown that a cavity containing a second-order nonlinear crystal can efficiently be used for the spectral power noise reduction of a continuous-wave laser beam of $0.75 \mathrm{~W}$ power within the bandwidth of the cavity. The cavity was built and operated such that it could act as an effective Kerr nonlinear device. The cavity was detuned from its resonance and the temperature of the crystal was set to a secondharmonic conversion minimum. We demonstrated a strong power noise reduction of $32 \mathrm{~dB}$ at a frequency corresponding to about $1 / 5$ of the cavity bandwidth and below. To the best of our knowledge, such a high value of noise suppression from the Kerr effect has not been reported before for continuous-wave laser light.

The noise reduction technique presented here can be combined with standard techniques such as passive filtering through mode cleaner cavities. But in particular for high laser powers and for amplitude noise at rather low Fourier frequencies, say the $\mathrm{kHz}$ regime and below, the technique investigated here proves to be rather powerful. It allows for a strong noise reduction at frequencies much smaller than the cavity linewidth, without photoelectric detection of the laser beam. In principle, the optical Kerr effect can even be used for a noise reduction at and below the shot noise level. In particular, for high-power continuous-wave lasers, this nonclassical regime of noise suppression remains challenging. Our future work aims for the noise suppression of the new generation of single-mode high-power lasers for advanced gravitational-wave detectors. These lasers provide powers of up to $200 \mathrm{~W}$. We expect that at such high powers, the noise reduction by means of a Kerr nonlinear cavity will prove to be even more powerful. Since a smaller cavity power buildup is required, the cavity finesse can be relaxed and optical losses thus be reduced.

\section{ACKNOWLEDGMENTS}

We would like to thank Albrecht Rüdiger for helpful and valuable remarks for the preparation of this paper. This work has been supported by the Deutsche Forschungsgemeinschaft and is part of Sonderforschungsbereich 407. A.K. was supported by the Studienstiftung des Deutschen Volkes.
[1] F. Seifert, P. Kwee, M. Heurs, B. Willke, and K. Danzmann, Opt. Lett. 31, 2000 (2006).

[2] P. Kwee, B. Willke, and K. Danzmann, Opt. Lett. 33, 1509 (2008).

[3] N. Imoto, H. A. Haus, and Y. Yamamoto, Phys. Rev. A 32, 2287 (1985).

[4] M. Kitagawa and Y. Yamamoto, Phys. Rev. A 34, 3974 (1986).

[5] H. Rehbein, J. Harms, R. Schnabel, and K. Danzmann, Phys. Rev. Lett. 95, 193001 (2005).

[6] K. Bergman and H. A. Haus, Opt. Lett. 16, 663 (1991).

[7] R. Dong, J. Heersink, J. F. Corney, P. D. Drummond, U. L. Andersen, and G. Leuchs, Opt. Lett. 33, 116 (2008).

[8] L. A. Ostrovskii, Zh. Eksp. Teor. Fiz. Pis'ma Red. 5, 331 (1967).
[9] A. G. White, P. K. Lam, D. E. McClelland, H.-A. Bachor and W. J. Munro, J. Opt. B: Quantum Semiclassical Opt. 2, 553 (2000).

[10] K. S. Zhang, T. Coudreau, M. Martinelli, A. Maitre, and C. Fabre, Phys. Rev. A 64, 033815 (2001).

[11] D. F. Walls and G. J. Milburn, Quantum Optics (Springer, New York, 2008).

[12] M. Shirasaki and H. A. Haus, J. Opt. Soc. Am. B 8, 681 (1991).

[13] A. G. White, J. Mlynek, and S. Schiller, Europhys. Lett. 35, 425 (1996).

[14] G. I. Stegeman, M. Sheik-Bahae, E. Van Stryland, and G. Assanto, Opt. Lett. 18, 13 (1993).

[15] P. Tombesi, Phys. Rev. A 39, 4288 (1989). 
[16] A. Berzanskis, K.-H. Feller, and A. Stabinis, Opt. Commun. 118, 438 (1995).

[17] R. W. Boyd, Nonlinear Optics (Academic Press, New York, 2003).

[18] H. J. Kimble, Y. Levin, A. B. Matsko, K. S. Thorne, and S. P. Vyatchanin, Phys. Rev. D 65, 022002 (2001).

[19] S. Chelkowski, H. Vahlbruch, B. Hage, A. Franzen, N.
Lastzka, K. Danzmann, and R. Schnabel, Phys. Rev. A 71, 013806 (2005).

[20] P. Kwee and B. Willke, Appl. Opt. 47, 6022 (2008).

[21] R. W. P. Drever, J. L. Hall, F. V. Kowalski, J. Hough, G. M. Ford, A. J. Munley, and H. Ward, Appl. Phys. B: Lasers Opt. 31, 97 (1983).

[22] N. Lastzka and R. Schnabel, Opt. Express 15, 7211 (2007). 